\title{
Activity of Tixagevimab/Cilgavimab against the Omicron variant of SARS-CoV-2 in a hamster model
}

\section{Jean-Sélim DRIOUICH}

Unité des Virus Émergents (UVE: Aix-Marseille Univ-IRD 190-INSERM 1207)

\section{Guillaume Lingas}

Université de Paris, IAME, INSERM, Paris, France

\section{Léa Luciani}

Unité des Virus Émergents (UVE: Aix-Marseille Univ-IRD 190-INSERM 1207)

Maxime Cochin

Unité des Virus Émergents (UVE: Aix-Marseille Univ-IRD 190-INSERM 1207)

\section{Paola Mariela Saba Villarroel}

Unité des Virus Émergents (UVE: Aix-Marseille Univ-IRD 190-INSERM 1207-IHU Méditerranée Infection) https://orcid.org/0000-0002-8695-5283

\section{Gregory Moureau}

Unite des Virus Emergents (UVE: Aix-Marseille Univ - IRD 190 - Inserm 1207 - IHU Mediterranee Infection), Marseille

\section{Paul Rémi Petit}

Unité des Virus Émergents (UVE: Aix-Marseille Univ-IRD 190-INSERM 1207)

\section{Franck Touret}

UVE Aix Marseille Univ, IRD 190, INSERM 1207, IHU Méditerranée Infection https://orcid.org/00000002-4734-2249

Jeremie Guedj

INSERM https://orcid.org/0000-0002-5534-5482

\section{Xavier de Lamballerie}

Aix Marseille Univ https://orcid.org/0000-0001-7895-2720

Antoine Nougairède ( $\square$ antoine.nougairede@univ-amu.fr)

Unité des Virus Émergents (UVE: Aix-Marseille Univ-IRD 190-INSERM 1207) https://orcid.org/00000001-7729-2636

\section{Brief Communication}

Keywords:

Posted Date: March 1st, 2022 
DOI: https://doi.org/10.21203/rs.3.rs-1399448/v1

License: (c) (i) This work is licensed under a Creative Commons Attribution 4.0 International License. Read Full License 


\title{
Activity of Tixagevimab/Cilgavimab against the Omicron variant of SARS-CoV-2 in a hamster model
}

Jean-Sélim Driouich ${ }^{1}$, Guillaume Lingas ${ }^{2}$, Léa Luciani ${ }^{1}$, Maxime Cochin ${ }^{1}$, Paola Mariela Saba Villarroel ${ }^{1}$, Grégory Moureau ${ }^{1}$, Paul-Rémi Petit ${ }^{1}$, Franck Touret ${ }^{1}$, Jérémie Guedj ${ }^{2}$, Xavier de Lamballerie ${ }^{1 \#} \&$ Antoine Nougairède $^{1 \#}$

1: Unité des Virus Émergents, UVE: Aix Marseille Univ, IRD 190, INSERM 1207, Marseille, France.

2: Université de Paris, IAME, INSERM, F-75018 Paris, France

\#: These authors contributed equally

\begin{abstract}
The efficacy of pre-exposure prophylaxis by the Tixagevimab/Cilgavimab cocktail (AZD7442) was evaluated in hamsters against a clinical BA.1 strain of SARS-CoV-2 variant Omicron. AZD7442 retains inhibitory activity against Omicron despite a substantial loss of efficacy. We estimate that Omicron virus requires about 20-times more antibodies in plasma than the ancestral B.1 strain (G614) virus to achieve a similar drug efficacy in reducing lung infectious titers.
\end{abstract}

\section{Main text}

Since its emergence in 2019, the severe acute respiratory syndrome coronavirus 2 (SARS-CoV-2), the causative agent of the coronavirus disease 2019 (COVID-19), has evolved rapidly, leading to the successive emergence of variants carrying mutations on the spike protein. Assessing the degree of escape of circulating variants from naturally produced antibodies (previous infections or after vaccination) or therapeutic antibodies is crucial to inform public health policies ${ }^{1}$.

The Omicron variant (lineage B.1.1.529) emerged in South Africa in late 2021 and has rapidly spread worldwide with very high incidence levels ${ }^{2}$. This variant contains a large number of mutations, including over 30 in the spike protein. Some of these mutations are associated with potential escape from humoral immunity and higher transmissibility ${ }^{3,4}$. Several in vitro studies have shown that monoclonal antibodies already used against SARS-COV-2 during the pandemic have reduced or no efficacy against the Omicron BA.1 variant ${ }^{5-10}$. In one of them, the activity of the combination of Cilgavimab/Tixagevimab (AZD7442) that targets the SARS-CoV-2 spike receptor binding domain (RBD) ${ }^{11}$ and marketed by AstraZeneca as Evusheld, was reduced by a factor of $\sim 40^{5}$.

Here, we evaluated the efficacy of AZD7442 on a clinical strain (2021/FR/1514) of the Omicron BA.1 variant using the hamster model and an ancestral B.1 (G614) strain as reference. Animals received pre-exposure prophylaxis by intraperitoneal injection of 2,4 or $16 \mathrm{mg} / \mathrm{kg}$ of AZD7442. Animals were infected intranasally $\left(3 \times 10^{4} \mathrm{TCID}_{50}\right)$ seven days later with Omicron variant or $\mathrm{G} 614$ strain, and sacrificed at 3 days post-infection (dpi) (figure 1a). 
Figure 1: In vivo efficacy of pre-exposure prophylaxis of AZD7442 on G614 strain and Omicron BA.1 variant

(a) Experimental timeline (realized on biorender.com). Groups of hamsters were intraperitoneally treated with 0 ( $\mathrm{n}=6$ ; untreated group), 2, 4 or $16 \mathrm{mg} / \mathrm{kg}$ of AZD7442 ( $\mathrm{n}=5$ per group). Seven days later, animals were intranasally infected with $3 \times 10^{4} \mathrm{TCID}_{50}$ of virus and sacrificed at 3 dpi. (b) Lung infectious titers measured using a TCID 50 assay. Viral RNA yields in lungs (c) and in nasal washes (d) measured using a RT-qPCR assay. (b-d) Data represent mean \pm SD of individual data of hamsters. ** and * symbols indicate that lung infectious titers, viral RNA yields in lungs/nasal washes are significantly smaller than those of the untreated group with a p-value ranging between 0.001-0.01 and 0.010.05 , respectively (Two-sided statistical analysis were performed using Shapiro-Wilk normality test, Student t-test, Mann-Whitney test, and Welch's test). (e) Dose-response curves based on lung infectious titers were established to determine 90\% effective concentrations in plasmas of animals (Animals infected with G614 strain or Omicron variant were represented in red and blue respectively). Clinical follow-up of this experiment is presented in Supplemental Figure 1.

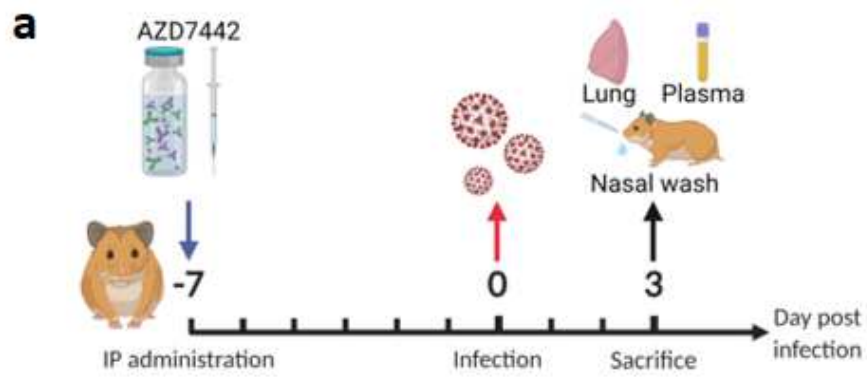

b

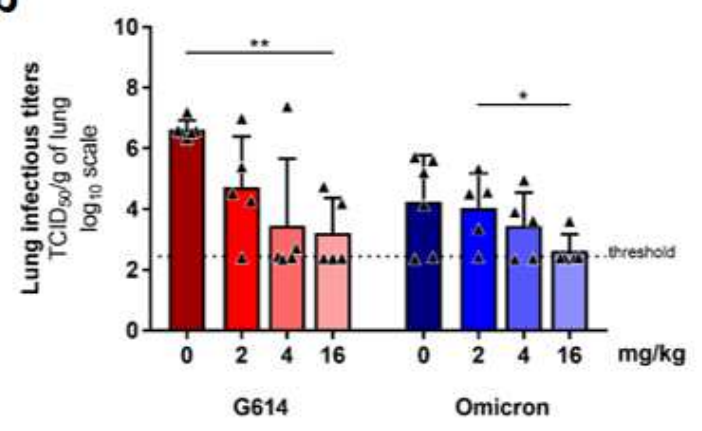

d

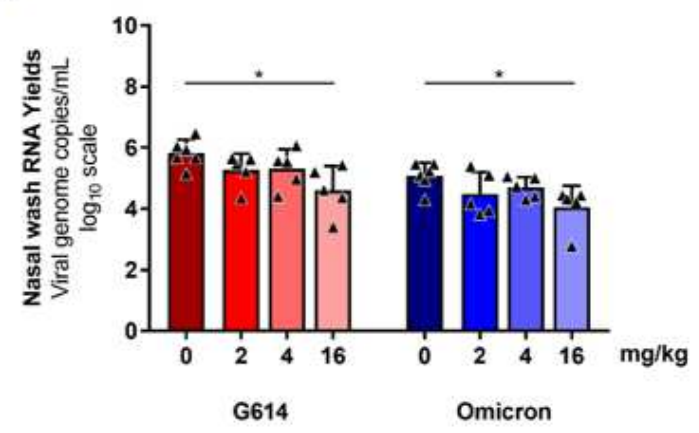

C
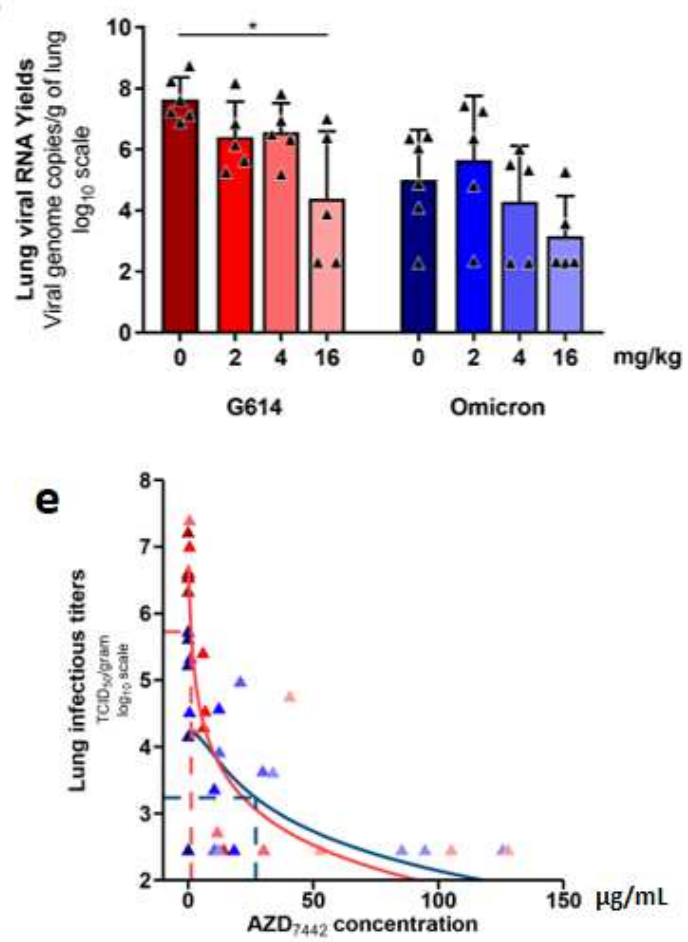
In animals infected with strain G614, administration of AZD7442 resulted in a dose-dependent decrease in infectious titers and viral RNA levels in the lungs (figure $\mathbf{1 b}-\mathbf{c}$ ). This decrease is significant with the dose of $16 \mathrm{mg} / \mathrm{kg}$ when compared with untreated animals ( $p \leq 0.0278$ ). With regard to viral load in nasal washes, a dose-dependent reduction in the amount of viral RNA is also observed but to a lesser extent than in the lungs. (figure 1d). It is significant with the higher dose when compared with untreated animals ( $p=0.0106)$. Regarding the impact of AZD7442 administration on the weight of the animals, we found that all doses induced a clinical attenuation of the disease at 3dpi (supplemental figure 1).

In animals infected with the Omicron variant, administration of AZD7442 also induced a dose-dependent reduction in infectious titers and viral RNA levels in the lungs (figure $\mathbf{1 b}-\mathbf{c}$ ). This decrease is significant for infectious titers at the dose of $16 \mathrm{mg} / \mathrm{kg}$ compared to animals treated at $2 \mathrm{mg} / \mathrm{kg}(p=0.0317)$. As with strain G614, a limited dose-dependent decrease is also observed in nasal washes (figure 1d). It is significant with the $16 \mathrm{mg} / \mathrm{kg}$ dose when compared with untreated animals $(p=0.0303)$. As Omicron infection has no detectable effect on the weight of the animals in our model, the impact of AZD7442 administration on the clinical course of the disease could not be assessed in this way. (supplemental figure 1) ${ }^{12}$.

Next, the concentration of AZD7442 in plasma was measured in animals sacrificed at 3 dpi. Variable exposures were observed for identical doses, which may be related to the intraperitoneal route of administration. This variability allowed investigating the relationship between animal exposure to AZD7442 and infectious viral loads in the lungs (figure 1e). We conducted a nonlinear regression of infectious viral titers against plasma concentration of AZD7442. It allowed estimating $90 \%$ effective concentrations $\left(\mathrm{EC}_{90}\right)$ in plasma to reduce lung infectious titers with each viral strain: $1.2 \mu \mathrm{g} / \mathrm{mL}(95 \%$ confidence interval $(95 \% \mathrm{Cl}): 0.7-1.7)$ and $27.0 \mu \mathrm{g} / \mathrm{mL}(95 \% \mathrm{Cl}: 0-54.7)$ for the $\mathrm{G} 614$ strain and the Omicron variant, respectively, indicating a 22 fold change between the two strains.

Overall, our results indicate that AZD7442, when used as pre-exposure prophylaxis, has inhibitory activity in vivo against the Omicron BA.1 variant, although this activity is less than against a B.1 G614 virus. This is in line with previous results obtained in vitro ${ }^{5-10}$ and a recent in vivo study in which a curative treatment with $5 \mathrm{mg} / \mathrm{kg}$ of antibody cocktail, synthesized without any modification in their Fc region, efficiently inhibited the Omicron variant replication in lungs of hamsters ${ }^{13}$. The U.S. Food Drug Administration (FDA) has published pharmacokinetic data obtained after intramuscular administration of 300mg of AZD7442 ${ }^{14}$, the dose used successfully in the PROVENT clinical trial (NCT04625725) ${ }^{15}$. The mean serum concentrations peak at $\sim 30 \mu \mathrm{g} / \mathrm{mL}$ two weeks after dosing and remain above $12 \mu \mathrm{g} / \mathrm{mL}$ for more than 150 days, with limited inter-individual variability (coefficient of variation was 25-39\% even beyond 200 days after dosing). In our study, the plasma $\mathrm{EC}_{90}$ value estimated from hamsters treated preventively and infected with the G614 strain are largely below these concentrations. In contrast, the $\mathrm{EC}_{90}$ value estimated in hamsters infected by Omicron is close to the abovementioned exposures observed in humans after the dose of $300 \mathrm{mg}$. However, the linear and dose-dependent pharmacokinetics of AZD7442 between the $300 \mathrm{mg}$ and 3000mg doses suggest that the $600 \mathrm{mg}$ dose, currently being tested in the TACKLE, ACTIV-2/3 and DisCoVeRy clinical trials (D8851C00001, NCT04723394, NCT04518410, NCT04501978 and NCT04315948), may be a recourse to compensate for the diminished efficacy of this monoclonal antibody cocktail against the Omicron variant. This increase in AZD7442 dosage has just been recommended by the U.S. FDA for pre-exposure prophylaxis of SARS-CoV-2 infection ${ }^{16}$. 


\section{References}

1 Murano, K., Guo, Y. \& Siomi, H. The emergence of SARS-CoV-2 variants threatens to decrease the efficacy of neutralizing antibodies and vaccines. Biochem Soc Trans 49, 2879-2890, doi:10.1042/BST20210859 (2021).

2 Viana, R. et al. Rapid epidemic expansion of the SARS-CoV-2 Omicron variant in southern Africa. Nature, doi:10.1038/s41586-022-04411-y (2022).

3 Karim, S. S. A. \& Karim, Q. A. Omicron SARS-CoV-2 variant: a new chapter in the COVID-19 pandemic. Lancet (London, England) 398, 2126-2128, doi:10.1016/S0140-6736(21)02758-6 (2021).

4 Kumar, S., Thambiraja, T. S., Karuppanan, K. \& Subramaniam, G. Omicron and Delta variant of SARS-CoV-2: A comparative computational study of spike protein. Journal of medical virology 94, 1641-1649, doi:10.1002/jmv.27526 (2022).

5 Touret, F., Baronti, C., Bouzidi, H. S. \& de Lamballerie, X. In vitro evaluation of therapeutic antibodies against a SARS-CoV-2 Omicron B.1.1.529 isolate. bioRxiv, 2022.2001.2001.474639, doi:10.1101/2022.01.01.474639 (2022).

6 Aggarwal, A. et al. SARS-CoV-2 Omicron: evasion of potent humoral responses and resistance to clinical immunotherapeutics relative to viral variants of concern. medRxiv, 2021.2012.2014.21267772, doi:10.1101/2021.12.14.21267772 (2021).

7 Planas, D. et al. Considerable escape of SARS-CoV-2 Omicron to antibody neutralization. Nature, doi:10.1038/s41586-021-04389-z (2021).

8 Cao, Y. et al. Omicron escapes the majority of existing SARS-CoV-2 neutralizing antibodies. Nature, doi:10.1038/s41586-021-04385-3 (2021).

9 Cameroni, E. et al. Broadly neutralizing antibodies overcome SARS-CoV-2 Omicron antigenic shift. Nature, doi:10.1038/s41586-021-04386-2 (2021).

10 VanBlargan, L. A. et al. An infectious SARS-CoV-2 B.1.1.529 Omicron virus escapes neutralization by therapeutic monoclonal antibodies. Nat Med, doi:10.1038/s41591-021-01678-y (2022).

11 Dong, J. et al. Genetic and structural basis for recognition of SARS-CoV-2 spike protein by a twoantibody cocktail. bioRxiv, 2021.2001.2027.428529, doi:10.1101/2021.01.27.428529 (2021).

12 McMahan, K. et al. Reduced Pathogenicity of the SARS-CoV-2 Omicron Variant in Hamsters. bioRxiv, 2022.2001.2002.474743, doi:10.1101/2022.01.02.474743 (2022).

13 Uraki, R. et al. Therapeutic efficacy of antibodies and antivirals against a SARS-CoV-2 Omicron variant, PREPRINT (Version 1) available at Research Square [https://doi.org/10.21203/rs.3.rs$1240227 / \mathrm{v} 1]$ (2022).

14 US Food and Drug Administration. Fact sheet for health care providers: Emergency Use Authorization for Evusheld (tixagevimab co-packaged with cilgavimab). https://www.fda.gov/media/154701/download. Accessed February 17, 2022 (2022). AstraZeneca. Evusheld long-acting antibody combination retains neutralizing activity against Omicron variant in independent FDA study. Published December 16, 2021.

https://www.astrazeneca.com/. Accessed February 21, 2022 (2022).

US Food and Drug Administration. FDA authorizes revisions to Evusheld dosing . https://www.fda.gov/drugs/drug-safety-and-availability/fda-authorizes-revisions-evushelddosing. Accessed February 26, 2022 (2022).

17 Driouich, J. S. et al. Favipiravir antiviral efficacy against SARS-CoV-2 in a hamster model. Nature communications 12, 1735, doi:10.1038/s41467-021-21992-w (2021). 


\section{Methods}

$\underline{\text { Cells }}$

VeroE6 cells (ATCC CRL-1586) were cultured at $37^{\circ} \mathrm{C}$ with $5 \% \mathrm{CO} 2$ in minimal essential medium (MEM) supplemented with $1 \%$ Penicillin/Streptomycin, $1 \%$ non-essential amino acids and $7 \%$ of heat-inactivated fetal bovine serum (FBS) (all from ThermoFisher Scientific). VeroE6/TMPRSS2 cells (NIBSC 100978) were cultured in the same medium supplemented with $2 \%$ of G-418 (ThermoFisher Scientific).

Viruses

The B.1 BavPat1 SARS-CoV-2 strain (G614 strain) was obtained from Pr. C. Drosten through EVA GLOBAL (https://www.european-virus-archive.com/) and contains the D614G mutation. Virus stocks of this strain was produced using VeroE6 cells (passage history: 2 for in vivo studies). The clinical strain of the SARSCoV-2 BA.1 Omicron variant used here is named 2021/FR/1514 and is available through EVA GLOBAL (www.european-virus-archive.com, ref: 001V-04436, GISAID: EPI_ISL_7899754). Virus stocks of this strain was produced using VeroE6/TMPRSS2 cells (passage history: 1 for in vivo studies). All virus stocks were characterized by whole-genome sequencing (Ion Torrent) in order to verify the absence of additional mutations, especially in the spike-coding region. All experiments with infectious viruses were performed in a biosafety level 3 laboratory.

Antibodies

The combination Cilgavimab/Tixagevimab (AZD7442, Evusheld, AstraZeneca) were obtained from pharmacy of the University hospital of La Timone, Marseille, France.

\section{$\underline{\text { In vivo experiments }}$}

All experiments were performed in accordance with national and European guidelines and covering the use of animals for scientific purposes and following an approval by the local ethical committee and the French 'Ministère de l'Enseignement Supérieur, de la Recherche et de l'Innovation' (APAFIS\#23975).

Animals were housed in ISOcage P - Bioexclusion System (Techniplast) with unlimited access to food/water and $14 \mathrm{~h} / 10 \mathrm{~h}$ light/dark cycle. The animals were monitored and weighed daily to detect the appearance of clinical signs of pain, suffering or distress. Infection, nasal washes and intramuscular administrations were performed under general anesthesia (isoflurane). Animals were infected with $3.10^{4} \mathrm{TCID}_{50}$ of virus (virus diluted in $0.9 \%$ sodium chloride solution, intranasal infection with $50 \mu \mathrm{L}$ ). Organs and blood were collected after euthanasia (cervical dislocation under general anesthesia (isoflurane)).

\section{Organ and blood collection}

Nasal washes were performed with $150 \mu \mathrm{l}$ of $0.9 \%$ sodium chloride solution and transferred into $1.5 \mathrm{~mL}$ tubes containing $0.5 \mathrm{~mL}$ of $0.9 \%$ sodium chloride solution, centrifuged at $16,200 \mathrm{~g}$ for 10 minutes and stored at $-80^{\circ} \mathrm{C}$. Lung and blood samples were taken just after the animals were sacrificed. Left pulmonary lobes were washed with $10 \mathrm{~mL}$ of $0.9 \%$ sodium chloride solution, blotted with filter paper, weighed, transferred into $2 \mathrm{~mL}$ tubes containing $1 \mathrm{~mL}$ of $0.9 \%$ sodium chloride solution and $3 \mathrm{~mm}$ glass beads, crushed using a Tissue Lyser machine (Retsch MM400) for 20min at $30 \mathrm{cycles} / \mathrm{s}$ and centrifuged $10 \mathrm{~min}$ at 16,200g. Supernatant media were transferred into $1.5 \mathrm{~mL}$ tubes, centrifuged $10 \mathrm{~min}$ at $16,200 \mathrm{~g}$ and stored at $-80^{\circ} \mathrm{C}$. Plasma collection: Recovery of plasma: One milliliter of blood was harvested in a $2 \mathrm{~mL}$ tube containing 
$100 \mu \mathrm{L}$ of $0.5 \mathrm{M}$ EDTA (ThermoFischer Scientific) and centrifuged $10 \mathrm{~min}$ at $16,200 \mathrm{~g}$. Recovery of serum: One milliliter of blood was harvested in a $2 \mathrm{~mL}$ tube incubate $15 \mathrm{~min}$ at room temperature and centrifuged $10 \mathrm{~min}$ at $16,200 \mathrm{~g}$. Blood-derived samples were stored at $-80^{\circ} \mathrm{C}$.

\section{$\underline{\mathrm{TCID}_{50}} \underline{\text { assay }}$}

Virus titration was performed with 96-well culture plates containing confluent VeroE6/TMPRSS2 cells inoculated with $150 \mu \mathrm{L}$ per well of four-fold dilutions of samples (dilutions with medium supplemented with $2.5 \%$ FBS). After 6 days of incubation the absence/presence of cytopathic effect in each well was read and infectious titers were estimated using the Reed \& Muench calculation method.

\section{Real time quantitative RT-PCR (RT-qPCR) assays}

For viral quantification in lungs clarified homogenates, nasal washes and plasma, nucleic acids from each sample were extracted using QIAamp 96 DNA kit and Qiacube HT robot (both from Qiagen). Viral RNA yields were measured using a real time RT-qPCR assay targeting the rdrp gene as previously described ${ }^{17}$.

\section{Quantification of Cilgavimab/Tixagevimab monoclonal antibodies in blood samples}

To estimate the exposure of animals to Cilgavimab/Tixagevimab monoclonal antibodies (AZD7442), we measured the level of human IgG antibodies directed against the S1 domain of the spike protein of the SARS-CoV-2 using a commercial enzyme-linked immunosorbent assay (ELISA) kit (Euroimmun). Results were expressed in binding antibody units per $\mathrm{mL}(\mathrm{BAU} / \mathrm{mL})$ following manufacturer instructions and converted to $\mu \mathrm{g} / \mathrm{mL}$ using blank plasma from untreated/infected animals spiked with known quantities of AZD7442.

\section{EC 90 determination}

We used the following non-linear function to determine the concentration/effect relationship:

$$
\mathrm{VL}=\mathrm{VL}_{0} \times\left(1-\frac{\mathrm{C}_{\mathrm{AZD7442}}^{\gamma}}{\mathrm{EC}_{50}^{\gamma}+\mathrm{C}_{\mathrm{AZD7442}}^{\gamma}}\right)
$$

Where $\mathrm{VL}_{0}$ is the lung viral load in untreated animals, $\mathrm{C}_{\mathrm{AZD7442}}$ is the plasma concentration of AZD$7442, \mathrm{EC}_{50}$ is the drug concentration required to decrease viral load by $50 \%$ compared to untreated animals, and $\gamma$ is a sigmoidicity parameter, chosen as the one maximizing likelihood of the model. We

extrapolated the $E C_{90}$ using $\mathrm{EC}_{90}=\sqrt[\gamma]{9 \times \mathrm{EC}_{50}^{\gamma}}$. Parameters were estimated separately according to strain, using non-linear regression. Values under the limit of detection (LoD) for lung infectious titers or AZD7442 concentrations were imputed to this LoD, respectively $2.43 \mathrm{TCID}_{50} / \mathrm{g}$ and $0.54 \mu \mathrm{g} / \mathrm{mL}$.

\section{Graphical representations and statistical analysis}

Experimental timelines were generated on biorender.com. Graphical representations and statistical analyses) were realized using Graphpad Prism 7 software, except nonlinear regressions and their corresponding graphical representations that were performed using the package $n / r e g$, implemented in $R$ statistical software (http://www.R-project.org). Statistical details for each experiment are provided in the figure legends. P values less than 0.05 were considered statistically significant. 


\section{Funding}

This work was conducted in the framework of the Preclinical Study Group of the French agency for emerging infectious diseases (ANRS-MIE). It was supported by the ANRS-MIE (BIOVAR project of the EMERGEN research programme) and by the European Commission (European Virus Archive Global project (EVA GLOBAL, grant agreement No 871029) of the Horizon 2020 research and innovation programme).

\section{Contribution}

AN and XDL conceived the experiments. XDL proposed the study. JSD, LL, MC, PMSV, GM, PRP, FT and AN performed the experiments. JSD, GL, LL, MC, JG and AN analyzed the results. JSD, AN and XDL wrote the paper. All the authors reviewed and edited the paper.

\section{Acknowledgments}

We thank Pr C Drosten for providing the SARS-CoV-2 BavPat strain through EVA GLOBAL.

\section{Competing interests}

The authors declare no competing interests. 


\section{Supplemental data}

Supplemental figure 1: Clinical follow-up of animals

A Normalized weights of animals infected by the G614 strain. B Normalized weights of animals infected by the Omicron variant. Normalized weight at day $n$ was calculated as follows: $\%$ of initial weight of the animal at day $\mathrm{n}$. Data represent mean $\pm \mathrm{SD} . * * * *, * *$ and $*$ symbols indicate that the average value for the group is significantly different from that of the untreated group with a $p$-value $<0.0001$, ranging between $0.001-$ 0.01 , and $0.01-0.05$, respectively.

A

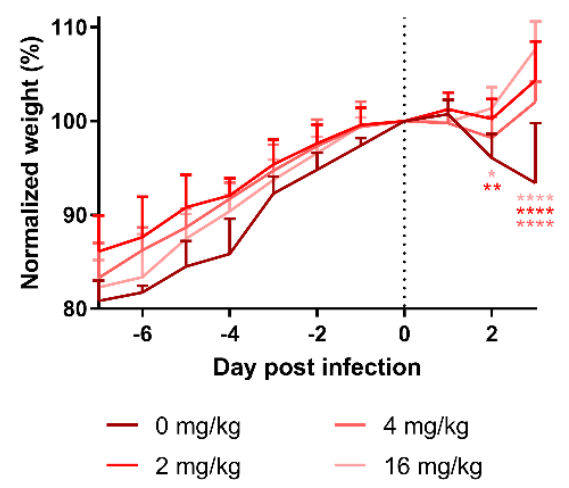

B

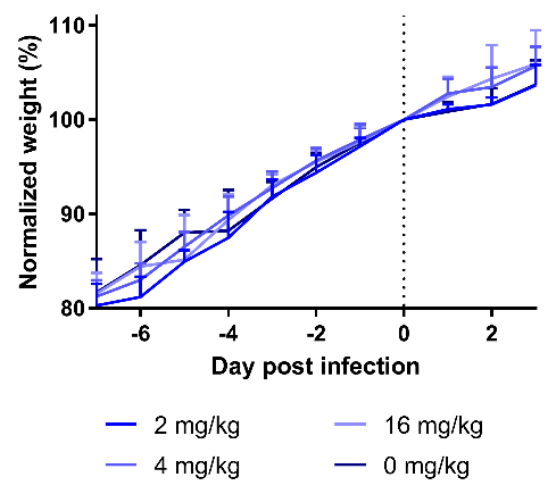

Supplemental figure 2: Plasma concentrations of AZD7442 after administration of single dose of AZD7442. AZD74442 concentrations were estimated using a commercial enzyme-linked immunosorbent assay that measures the level of human IgG antibodies directed against the S1 domain of the spike protein of the SARS-CoV-2.

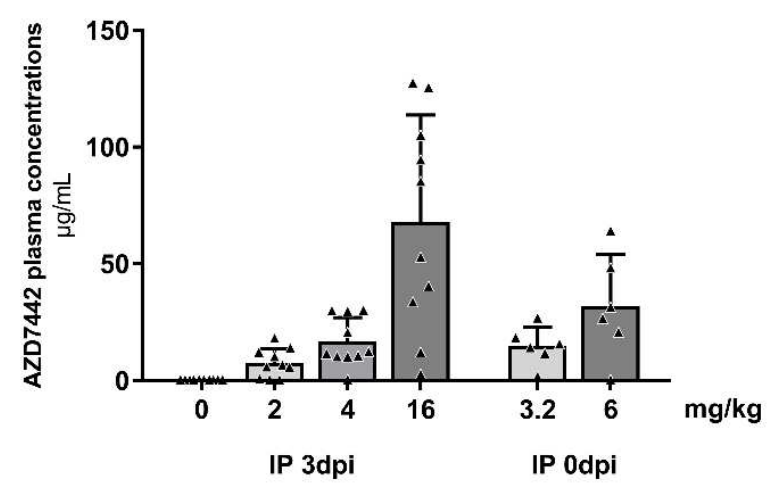

